\title{
Well-posedness for a class of fourth order diffusions for image processing
}

\author{
Patrick Guidotti and Kate Longo
}

\begin{abstract}
A number of image denoising models based on higher order parabolic partial differential equations (PDEs) have been proposed in an effort to overcome some of the problems attendant to second order methods such as the famous Perona-Malik model. However, there is little analysis of these equations to be found in the literature. In this paper, methods of maximal regularity are used to prove the existence of unique local solutions to a class of fourth order PDEs for noise removal. The proof is laid out explicitly for two newly proposed fourth order models, and an outline is given for how to apply the techniques to other proposed models.
\end{abstract}

Mathematics Subject Classification (2000). 35K35, 35K59, 35K65, 35Q68.

Keywords. Image processing noise removal, Fractional derivatives, Fourth order, Well-posedness, Nonlinear diffusions, Local existence.

\section{Introduction}

\subsection{Second order diffusions and regularizations}

Use of nonlinear PDEs for image denoising dates back to 1990 when Perona and Malik [30] proposed a dramatic change in the scale space diffusion method standard for smoothing noisy images. The scale space method, introduced by Witkin in 1983 [34], smoothes an image by convolving it with Gaussian kernels on a scale of variances. Equivalently, the smoothed images may be viewed as solution of the linear heat equation

$$
u_{t}=\gamma \Delta u, \quad u(0)=u_{0} \text { (original image), }
$$

where the diffusion coefficient $\gamma$ is constant. While this is an effective denoising method, it is ignorant of features in the image that one may wish to preserve; that is, it cannot distinguish noise from edges, resulting in the whole image becoming blurred. This method then needs to be complemented by a second processing step to locate and reintroduce edges. Perona and Malik's 
idea was to remove noise and preserve edges in a single step by replacing $\gamma$ with a nonlinear diffusivity which would inhibit diffusion across edges. This can be accomplished by observing that edges in an image correspond to regions of high gradient. Thus Perona and Malik consider $\gamma=g\left(|\nabla u|^{2}\right)$, where $g(\cdot)$ is chosen appropriately so as to slow diffusion (become small) when the edge detector $|\nabla u|^{2}$ is large. One possibility for such a function suggested by Perona and Malik is:

$$
g\left(s^{2}\right)=\frac{1}{1+c^{2} s^{2}}, \quad \text { for a constant } c>0 .
$$

In their paper, Perona and Malik propose discrete equations which can be interpreted as discretizations of the second order parabolic PDE

$$
u_{t}-\nabla \cdot\left(g\left(|\nabla u|^{2}\right) \nabla u\right)=0 .
$$

Such discretizations are effective at removing noise and preserving edges, but they exhibit a couple of notable shortcomings. They can create false edges, a phenomenon known as staircasing, as well as a blocky, cartoonish appearance in the smoothed image. Computing the divergence in 2 with the choice of $g$ given above (with $c=1$ ) yields

$$
\begin{aligned}
\nabla \cdot\left(\frac{1}{1+|\nabla u|^{2}} \nabla u\right) & =\frac{1}{1+|\nabla u|^{2}}\left[\Delta u-2 \frac{\nabla u^{T} D^{2} u \nabla u}{1+|\nabla u|^{2}}\right] \\
& =\frac{1}{1+|\nabla u|^{2}}\left[\partial_{\tau}^{2} u+\left(1-2 \frac{|\nabla u|^{2}}{1+|\nabla u|^{2}}\right) \partial_{\nu}^{2} u\right],
\end{aligned}
$$

where $\nu=\nabla u /|\nabla u|$ and $\tau$ is a unit vector orthogonal to $\nu$. Thus diffusion in the direction of $\nu$ may change sign in regions of steep gradient, resulting in backward diffusion, which is known to cause unstable behavior. This unstable behavior manifests itself as staircasing in numerical implementations. The forward-backward nature of 2 is exploited in [25] to prove its ill-posedness.

Perona and Malik suggested the diffusivity 1 in [30] with the idea that the backward diffusion across gradients would cause the sharpening of edges. (An alternate diffusivity with similar behavior, $g\left(s^{2}\right)=e^{-c^{2} s^{2}}$, is also suggested.) Indeed, the best feature of the Perona-Malik model, its edge detection capability, is the source of its ill-posedness. Many authors have searched for regularizations of 2 capable of preventing backward diffusion, thus leading to well-posedness, while still preserving edges. Charbonnier, et. al. [11] propose in the less degenerate diffusivity function,

$$
g\left(s^{2}\right)=\frac{1}{\sqrt{1+c^{2} s^{2}}},
$$

which does not lead to backward diffusion. See [15] for a more thorough discussion of different choices for diffusivities.

Others have suggested a regularization of the edge detector $|\nabla u|$, the argument inside the diffusivity function. Particularly novel and effective regularizations involving fractional derivatives are proposed by Guidotti and Lambers in [18-20]. Their proposed models are based on the equations

$$
u_{t}-\nabla \cdot\left(g\left(\left|\nabla^{1-\varepsilon} u\right|^{2}\right) \nabla u\right)=0
$$


and

$$
u_{t}-g\left(\left[(-\Delta)^{1-\varepsilon}\right]^{2}\right) \Delta u=0,
$$

coupled with appropriate initial and boundary conditions, where $\varepsilon \in(0,1)$. Fractional derivatives have been shown to be an effective tool for both regularization [14] and edge detection [29]. Indeed, it is shown in [18,19] that 3 and 4 are locally well-posed and that 3 admits characteristic functions of smooth sets as stationary solutions. Additionally, numerical experiments suggest that these two models produce significantly less staircasing than does 2 or other second order diffusion equations.

The use of fractional derivatives as an edge detection tools has proven more robust in the presence of noise (cf. [27,29]). This is most likely due to the fact that the corresponding kernels, while still singular, are non-local and thus provide some degree of averaging. The use of the Laplacian or its fractional powers as an edge detector is sensible for very sharp edges when the maximal curvature is observed very close to the edge itself while would become more debatable for less pronounced edges where maximal curvature would be dislocated as compared to the site the edge.

\subsection{Fourth order diffusions}

Another line of research applies the ideas of Perona and Malik to higher order equations in an effort to reduce staircasing and cartoonish effects. Equation 2 can be shown to be associated with the minimization of a first order energy functional. Many authors have considered instead second order energy functionals. Examples of such higher order variational methods can be found in $[5,9,10,26,28]$. A fourth order PDE associated with a second order energy functional is that proposed by You and Kaveh [35],

$$
u_{t}+\Delta\left(g\left((\Delta u)^{2}\right) \Delta u\right)=0 .
$$

Tumblin and Turk consider in [32] a fourth order PDE model designed to simplify an image based on its curvature,

$$
u_{t}+\nabla \cdot\left(g\left(\frac{1}{2}\left(u_{x x}^{2}+u_{y y}^{2}\right)+u_{x y}^{2}\right) \nabla \Delta u\right)=0 .
$$

Bertozzi and Greer propose in [6] a modification of 6 ,

$$
u_{t}+\nabla \cdot\left(g\left((\Delta u)^{2}\right) \nabla \Delta u\right)=0
$$

A high order generalization of Perona-Malik considered by Wei in [33] reads

$$
u_{t}+\nabla \cdot\left(g\left(|\nabla u|^{2}\right) \nabla \Delta u\right)=0 .
$$

Hajiaboli proposes in [22] a modification of 5 using $|\nabla u|$ as an edge detector, similar to

$$
u_{t}+\Delta\left(g\left(|\nabla u|^{2}\right) \Delta u\right)=0
$$

but with also a modified diffusivity function $g$. The same author considers another modification of 5 in [23] which utilizes an anisotropic diffusion tensor. 
The authors propose in [21] two modifications of 5 in which the edge detector is replaced with the fractional gradient used in 3 and the fractional Laplacian operator used in 4 , respectively. These two new equations read

$$
u_{t}+\Delta\left(g\left(\left|\nabla^{1-\varepsilon} u\right|\right) \Delta u\right)=0
$$

and

$$
u_{t}+\nabla \cdot\left(g\left((-\Delta)^{1-\varepsilon} u\right) \nabla \Delta\right)=0 .
$$

A widely used heuristic argument for the use of fourth order models is that they would avoid staircasing by virtue of having "affine functions" as stationary solutions (in contrast to some second order models where piecewise constant functions can be shown to be equilibria [18]). A rigorous analysis of the structure of the set of equilibria for fourth models is an interesting open question that is not addressed in this paper.

Finally we refer to [7] for a survey of other denoising methods, including those not based on PDEs.

\subsection{Well-posedness}

Denoising experiments performed with some of the above equations (see, in particular, $[15,21-23,28,35])$ show that fourth order models can avoid the staircasing and cartoonish effects of second order models while still removing noise and preserving edges. However, little analysis has been conducted on these fourth order equations.

In [17], Greer and Bertozzi prove the well-posedness of regularizations of 6 and 5. This result is in the same spirit as the many results regarding well-posedness of regularizations of the Perona-Malik equation, cf. $[3,8,18]$, for example.

Bertozzi and Greer address in [6] the existence of solutions to a special case of 7 . Their work relies on the structure of the particular choice of diffusivity function $g$, and is therefore difficult to generalize.

Didas, Weickert, and Burgeth, in [15], show that higher order generalizations of 2 such as 5 are $L_{2}$-stable and preserve average grey value, as well as higher order moments. The proofs in [15] can be easily modified to apply to 10 and 11 with appropriate boundary conditions. Since there is generally no maximum principle for fourth (or higher) order diffusions, it is not expected, or at least not obvious, that fourth order equations would be $L_{\infty}$-stable.

The effects of different choices of diffusivity function on 5 are also discussed in [15]. It is observed that the Perona-Malik diffusivity 1 promotes forward-backward diffusion, and consequently the sharpening of edges, in 5 as it does in 2. Indeed, expanding out the Laplacian term in 5 with $g\left(s^{2}\right)=$ $\left(1+s^{2}\right)^{-1}$ reveals that

$$
\begin{aligned}
\Delta\left(\frac{1}{1+(\Delta u)^{2}} \Delta u\right)= & \frac{1}{1+(\Delta u)^{2}}\left(1-\frac{2(\Delta u)^{2}}{1+(\Delta u)^{2}}\right) \Delta^{2} u \\
& +[\text { lower order terms }],
\end{aligned}
$$


and so backward diffusion can occur when $|\Delta u|$ is large. This likely implies that 5 , like 2 , is ill-posed. Unlike in 2, this backward diffusion is not necessarily in a direction perpendicular to edges, so in the fourth order case it is not obvious that backward diffusion contributes to edge sharpening as it does in the second order case.

Replacing the edge detector $|\Delta u|$ in 5 with $|\nabla u|$, as used in 2, eliminates this backward behavior. Expanding the Laplacian term of 9 in one dimension shows

$$
\begin{aligned}
\left(g\left(u_{x}^{2}\right) u_{x x}\right)_{x x}= & g\left(u_{x}^{2}\right) u_{x x x x} \\
& +\left[4 g^{\prime \prime}\left(u_{x}^{2}\right) u_{x}^{2} u_{x x}^{2}+2 g^{\prime}\left(u_{x}^{2}\right) u_{x x}^{2}+6 g^{\prime}\left(u_{x}^{2}\right) u_{x} u_{x x x}\right] u_{x x} .
\end{aligned}
$$

For any positive function $g$, the fourth order diffusion term never changes sign, and experiments show that with $g$ as in 1 the second order term does not have a consistent sign. This suggests that forward-backward diffusion is not necessary for good edge detection, and that 9 is well-posed.

This paper addresses the well-posedness of two new denoising models proposed in [21]. While the analysis is focused on equations 10 and 11, it can be easily modified to apply to every other equation listed in the previous section, with the exception of 5 . A theorem regarding the existence of local solutions to the new models is stated and proved in Sect. 3. The proof uses techniques from the theory of maximal regularity, which does not require the validity of a maximum principle, and allows for enough flexibility to work with nonlocal operators such as fractional derivatives and to apply the results to a large class of equations. Key results from this theory which are used in the proof are given in the appendices. It should be observed that the well-posedness results come at a price. While in applications one would like to allow for $\mathrm{L}_{\infty}$ initial data, the techniques used here require smoother initial data. Such assumptions could be somewhat relaxed if weak formulations were to be used instead, but bounded measurable data could only be treated by approximating them by smooth ones and then going to the limit by means of any available a priori estimate.

\section{New models}

We address the well-posedness of two modifications of the fourth order equation 5 proposed by You and Kaveh. One uses fractional derivatives to regularize the edge detector $\Delta u$ in 5 , and the other uses a fractional gradient as an edge detector, serving as a regularization of Eq. 9. Both Eqs. 5 and 9 can benefit from the regularizing properties of fractional derivatives. 5 becomes well-posed when its edge detector $|\Delta u|$ is raised to a fractional power, and the fractional power on the edge detector $|\nabla u|$ in 9 alleviates a numerical artifact which is observed under certain parameters. The denoising effects of these two models are illustrated in Figure 1.

Theorem 1 states the existence of local solutions to Eqs. 10 and 11. These equations are considered with periodic boundary conditions on a unit square, as this proved more effective in numerical experiments; Neumann conditions, 


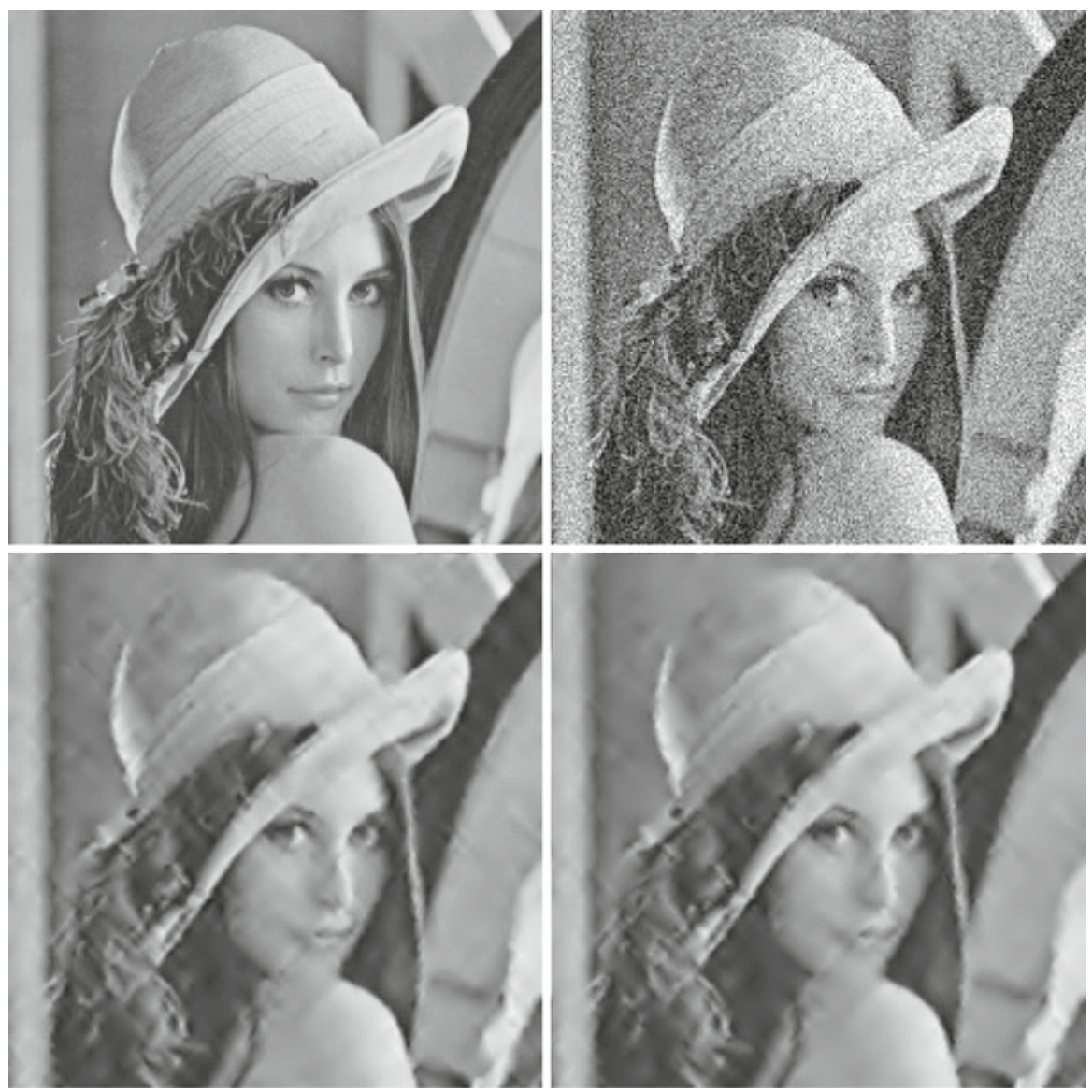

Figure 1. The denoising effects of Eqs. 12 and 13, with $g$ as in 1 and $\varepsilon=0.1$. Top row: clean image (left); image corrupted with $20 \%$ Gaussian noise (right). Bottom row: image denoised with $12, \varepsilon=0.1$ (left); image denoised with $13, \varepsilon=0.1$ (right).

on an arbitrary bounded domain with Lipschitz boundary, could also be considered, and the following theorem and proof could be reformulated to apply to that situation. The full equations being considered are

$$
\begin{cases}u_{t}+\Delta\left(g\left(\left|\nabla^{1-\varepsilon} u\right|^{2}\right) \Delta u\right)=0, & \text { in } \Omega \text { for } t>0, \\ u \text { periodic, } & \text { for } t>0, \\ u(0)=u_{0}, & \text { in } \Omega \text { for } t=0,\end{cases}
$$

and

$$
\begin{cases}u_{t}+\Delta\left(g\left(\left[(-\Delta)^{1-\varepsilon} u\right]^{2}\right) \Delta u\right)=0, & \text { in } \Omega \text { for } t>0, \\ u \text { periodic }, & \text { for } t>0, \\ u(0)=u_{0}, & \text { in } \Omega \text { for } t=0 .\end{cases}
$$


This theorem is particularly powerful in that its proof does not depend on the choice of diffusivity function $g$, except for requiring that this function be smooth and positive. The theorem can also be easily modified to accommodate a nonzero fidelity term, i.e.,

$$
u_{t}+\Delta\left(g\left(\left|\nabla^{1-\varepsilon} u\right|^{2}\right) \Delta u\right)=\lambda\left(u_{0}-u\right)
$$

provided it is sufficiently regular (see Theorem 3 and the accompanying discussion in appendix A). Such a fidelity term is often applied to insure that the smoothed image is not too dissimilar to the initial image. Numerical experiments on models 12 and 13 suggest, however, that such a term does not improve performance. Additionally, the techniques used in the proof of the theorem can be modified to be applied to equations of the form 6, 7, or 8. Consequently, Theorem 1 can be applied to a wide range of fourth order denoising models.

\section{Main theorem}

Theorem 1. Let $\Omega=[0,1]^{n}$, fix $\varepsilon \in(0,1)$ and $p>(4+n) / 2 \varepsilon$, and let $g \in$ $B U C^{\infty}([0, \infty),(0, \infty))$. For any $u_{0} \in W_{p, \pi}^{4-4 / p}(\Omega)$ there exists $T>0$ such that Eqs. 12 and 13 each possess a unique solution $u$ on $[0, T)$ satisfying

$$
u \in W_{p}^{1}\left(0, T ; L_{p}(\Omega)\right) \cap L_{p}\left(0, T ; W_{p, \pi}^{4}(\Omega)\right) .
$$

Remarks. (a) For Eq. 12, the theorem can be extended to allow $\varepsilon \in(-1,1)$ (thereby encompassing Eq. 9) with the condition that $p>(4+n) /(1+\varepsilon)$.

(b) Initial conditions are taken in Slobodeckii spaces. These spaces are defined in the next section. For simplicity of notation, the dependence of these spaces on $\Omega$ will be dropped except for when not clear from the context.

Theorem 1 is proved using techniques from the theory of maximal regularity. Background for the theory is postponed to appendix A, but some necessary preliminary definitions and results are given below.

\subsection{Function spaces and fractional derivatives}

Let the usual Sobolev norm be defined by

$$
\|u\|_{W_{p}^{k}}(\Omega):=\|u\|_{W_{p}^{k}}:=\left(\sum_{|\alpha| \leq k}\left\|\partial^{\alpha} u\right\|_{L_{p}(\Omega)}^{p}\right)^{1 / p}, \quad k \in \mathbb{N},
$$

where $\alpha \in \mathbb{N}^{n}$ is a multiindex, and define

$$
[u]_{s, p}:=\left(\int_{\Omega \times \Omega} \frac{|u(x)-u(y)|^{p}}{|x-y|^{n+s p}} d(x, y)\right)^{1 / p}, \quad 0<s<1 .
$$

Then for $s \in \mathbb{R}^{+} / \mathbb{N}$, the Slobodeckii spaces are the Banach spaces defined by

$$
W_{p}^{s}:=W_{p}^{s}(\Omega):=\left\{u \in W_{p}^{\lfloor s\rfloor}:[u]_{s, p}<\infty\right\},
$$

equipped with the norm 


$$
\|u\|_{W_{p}^{s}}:=\left(\|u\|_{W_{p}^{\lfloor s\rfloor}}^{p}+\sum_{|\alpha|=\lfloor s\rfloor}\left[\partial^{\alpha} u\right]_{s-\lfloor s\rfloor, p}^{p}\right)^{1 / p},
$$

where $\lfloor s\rfloor$ denotes the greatest integer less than or equal to $s$. Furthermore, for $s>0$, define

$$
W_{p, \pi}^{s}:=W_{p, \pi}^{s}(\Omega):=\left\{u \in W_{p}^{s}: u \text { is periodic on } \Omega\right\},
$$

and observe that $L_{p}=L_{p, \pi}$.

The fractional gradient, under periodic boundary conditions, can be defined by first observing that

$$
\partial_{z}=\mathcal{F}_{z}^{-1} \operatorname{diag}\left[(2 \pi i k)_{k \in \mathbb{Z}}\right] \mathcal{F}_{z},
$$

where $\mathcal{F}_{z}$ is the partial Fourier transform with respect to $z=x, y$. Then the fractional partial derivative is defined by

$$
\partial_{z}^{\rho}=\mathcal{F}_{z}^{-1} \operatorname{diag}\left[\left((2 \pi k)^{\rho} e^{i \rho \frac{\pi}{2} \operatorname{sign}(k)}\right)_{k \in \mathbb{Z}}\right] \mathcal{F}_{z},
$$

for $\rho \in \mathbb{R}^{+}$. The fractional gradient is finally given by

$$
\nabla^{\rho}=\left[\begin{array}{l}
\partial_{x}^{\rho} \\
\partial_{y}^{\rho}
\end{array}\right]
$$

Exponents of the positive definite operator $-\Delta$ with periodic boundary conditions can be defined through its symbol:

$$
(-\Delta)^{\rho}=\mathcal{F}^{-1} \operatorname{diag}\left[\left(4 \pi^{2}|k|^{2 \rho}\right)_{k \in \mathbb{Z}^{2}}\right] \mathcal{F},
$$

for $\rho \in \mathbb{R}^{+}$.

Since we are working with operators involving fractional derivatives, it is useful to have the Bessel potential spaces $H_{p, \pi}^{s}$ available. For $p \in(1, \infty), s>0$, define

$$
H_{p, \pi}^{s}:=H_{p, \pi}^{s}(\Omega):=\left\{u \in L_{p}:\|u\|_{H_{p}^{s}}<\infty\right\}
$$

where

$$
\|u\|_{H_{p}^{s}}:=\left\|\mathcal{F}^{-1} \operatorname{diag}\left[\left(\left(1+|k|^{2}\right)^{s / 2}\right)_{k \in \mathbb{Z}^{n}}\right] \mathcal{F} u\right\|_{L_{p}} .
$$

Fractional derivatives behave nicely on these spaces. It follows easily from the definitions that for $\rho>0, s \in \mathbb{R}, p \in(1, \infty)$, and $j=1, \ldots, n$,

$$
\partial_{x_{j}}^{\rho} \in \mathcal{L}\left(H_{p, \pi}^{s}, H_{p, \pi}^{s-\rho}\right)
$$

and

$$
(-\Delta)^{\rho} \in \mathcal{L}\left(H_{p, \pi}^{s}, H_{p, \pi}^{s-2 \rho}\right) .
$$

Furthermore, Slobodeckii spaces can be embedded into Bessel spaces. It follows from results in [1, Chapter 5], along with [31, Theorems 1.3.3(e) and 1.10.3], that for any $s>\delta>0$,

$$
W_{p, \pi}^{s} \hookrightarrow H_{p, \pi}^{s-\delta}
$$

and by a classical embedding theorem [31, Theorem 4.6.1(e)],

$$
H_{p, \pi}^{s} \hookrightarrow C^{s-n / p}(\bar{\Omega}),
$$

for $s>n / p$. 
The previous results imply that

$$
\partial_{x_{j}}^{\rho} \in \mathcal{L}\left(W_{p, \pi}^{s}, C^{s-\rho-(\delta+n / p)}(\bar{\Omega})\right),
$$

and

$$
(-\Delta)^{\rho} \in \mathcal{L}\left(W_{p, \pi}^{s}, C^{s-2 \rho-(\delta+n / p)}(\bar{\Omega})\right),
$$

whenever the exponents on the $C$ spaces are positive.

\subsection{Main lemma}

The backbone of the proof of Theorem 1 is the following lemma, which is an amalgam of several results from the theory of maximal regularity. The proof of the lemma is postponed to appendix A.

Lemma 2. Let $p \in[2, \infty)$ and $\Omega$ and $u_{0}$ as in Theorem 1 , and consider the equation

$$
\begin{cases}u_{t}+A(u) u=0, & \text { in } \Omega \text { for } t>0 \\ u \text { periodic, } & \text { for } t>0, \\ u(0)=u_{0}, & \text { in } \Omega \text { for } t=0\end{cases}
$$

where $A$ is a fourth order elliptic nonlinear differential operator. Suppose that there exists a nonempty open subset $U \subset W_{p, \pi}^{4-4 / p}$ containing $u_{0}$ such that the following hold:

(R1) $[v \mapsto A(v)] \in C^{1-}\left(U ; \mathcal{L}\left(W_{p, \pi}^{4}, L_{p}\right)\right) ;$

(R2) for any $v \in U$ there exist $M>0$ and $\theta \in[0, \pi / 2)$ such that $A(v)$ is a uniformly $(M, \theta)$-elliptic operator; and

(R3) for any $v \in U$ there exists $\rho \in(0,1)$ such that the coefficients of $A(v)=$ $\sum_{|\alpha| \leq 4} a_{\alpha} \partial^{\alpha}$ are Hölder continuous with exponent $\rho$, that is, for all $|\alpha| \leq 4, a_{\alpha} \in B U C^{\rho}(\Omega)$.

Then there exist $T>0$ and a unique function

$$
u \in W_{p}^{1}\left(0, T ; L_{p}\right) \cap L_{p}\left(0, T ; W_{p, \pi}^{4}\right)
$$

satisfying 16 for $t \in[0, T)$.

Remarks. The concept of a uniformly $(M, \theta)$-elliptic operator is defined in 29 in appendix $B$.

\subsection{Existence proof}

Proof of Theorem 1. Let $\mathrm{A} u$ denote either $\left|\nabla^{1-\varepsilon} u\right|$ or $(-\Delta)^{1-\varepsilon} u$, according to whether we are considering 12 or 13 . Let $U$ be a bounded open subset of $W_{p, \pi}^{4-4 / p}$ containing $u_{0}$. The operator of concern is

$$
A(u) u=\Delta\left[\Phi_{u} \Delta u\right]=\Phi_{u} \Delta^{2} u+\sum_{i=1}^{n}\left(\partial_{x_{i}} \Phi_{u}\right)\left(\partial_{x_{i}} \Delta u\right)+\left(\Delta \Phi_{u}\right)(\Delta u)
$$

with

$$
\Phi_{u}=g\left((\mathrm{~A} u)^{2}\right)
$$


Let $v \in W_{p, \pi}^{4-4 / p}$ be any. Then

$$
A(v) u=\Delta\left[\Phi_{v} \Delta u\right]=\sum_{|\alpha| \leq 4} a_{\alpha}(x) \partial^{\alpha} u,
$$

where $a_{\alpha}=a_{\alpha, v}: \Omega \mapsto \mathbb{C}$. (The $a_{\alpha}$ also depend on $v$, but for simplicity that dependence will be dropped from the notation when it is clear from the context.) When $n=2$,

$$
a_{\alpha}= \begin{cases}\Phi_{v}, & \alpha=(4,0),(0,4) \\ 2 \Phi_{v}, & \alpha=(2,2) \\ 2 \partial_{x_{1}} \Phi_{v}, & \alpha=(3,0),(1,2) \\ 2 \partial_{x_{2}} \Phi_{v}, & \alpha=(2,1),(0,3) \\ \Delta \Phi_{v}, & \alpha=(2,0),(0,2) \\ 0, & \text { otherwise. }\end{cases}
$$

(A similar calculation can be done when $n \neq 2$.) To prove the theorem, it suffices to show that $A$ satisfies (R1), (R2), and (R3) from Lemma 2.

(R3): Given the calculation above, it is sufficient to show that there is $\rho \in(0,1)$ such that $\Phi_{v} \in C^{2+\rho}(\bar{\Omega})$. 14, 15, and the condition on $p$ allow us to find $\rho \in(0,1)$ such that

$$
(\mathrm{A} v)^{2} \in C^{2+\rho}(\bar{\Omega})
$$

for either choice of $A$. It is discussed in [12] that the map

$$
w \mapsto f(w), C^{\beta}(\bar{\Omega}) \rightarrow C^{\beta}(\bar{\Omega})
$$

is, for any $\beta \in(0,1)$, well-defined, bounded, and uniformly Lipschitz continuous on bounded sets provided that the map $[x \mapsto f(x)]$ is twice continuously differentiable on its domain. Since $g \in B U C^{\infty}([0, \infty))$, it follows from this result and 17 that

$$
g\left((\mathrm{~A} v)^{2}\right), g^{\prime}\left((\mathrm{A} v)^{2}\right), \quad \text { and } \quad g^{\prime \prime}\left((\mathrm{A} v)^{2}\right) \in C^{\rho}(\bar{\Omega}) .
$$

Hölder spaces are algebras, so (R3) follows from 17 and 18.

(R2): The principal symbol of the operator $A(v)$ is given by

$$
\begin{aligned}
\mathcal{A}_{\pi}(x, \xi) & =\sum_{|\alpha|=4} a_{\alpha}(x)(i \xi)^{\alpha} \\
& =\Phi_{v}\left(\xi_{1}^{4}+2 \xi_{1}^{2} \xi_{2}^{2}+\xi_{2}^{4}\right) \\
& =\Phi_{v}|\xi|^{4} .
\end{aligned}
$$

$\Phi_{v}$ is positive by assumption, so in light of 17 , there exist $c, C>0$ such that

$$
0<c<\mathcal{A}_{\pi}(x, \xi)=\Phi_{v}(x)<C, \quad(x, \xi) \in \Omega \times S^{n-1} .
$$

Thus $\{\lambda \in \mathbb{C}: \operatorname{Re}(\lambda)<c\} \subset \rho\left(\mathcal{A}_{\pi}\right)$, and so because $\mathcal{A}_{\pi}$ is real, there is $\theta \in(0, \pi / 2)$ such that

$$
\sigma\left(\mathcal{A}_{\pi}\right) \subset\{\lambda \in \mathbb{C}: \operatorname{Re}(\lambda) \geq c\} \cap \mathbb{R} \subset \Sigma_{\theta}:=\{z \in \mathbb{C}:|\arg z| \leq \theta\} .
$$

It is also evident by 18 that there exists $M \geq C>0$ such that

$$
\max _{|\alpha|=4}\left\|a_{\alpha}\right\|_{\infty} \leq M
$$


Thus $A(v)$ satisfies the condition of $(M, \theta)$-ellipticity 29, and so (R2) holds.

(R1): Pick $v, w \in U \subset W_{p, \pi}^{4-4 / p}$. Then

$$
\begin{aligned}
& \|A(v)-A(w)\|_{\mathcal{L}\left(W_{p, \pi}^{4}, L_{p}\right)} \\
& \quad=\sup _{\|u\|_{W_{p, \pi}^{4}}^{4}=1}\|(A(v) u-A(w)) u\|_{L_{p}} \\
& \quad \leq \sum_{|\alpha| \leq 4} \sup _{\|u\|_{W_{p, \pi}^{4}}=1}\left\|\left(a_{\alpha, v}-a_{\alpha, w}\right) \partial^{\alpha} u\right\|_{L_{p}} \\
& \quad \leq \sum_{|\alpha| \leq 4} \sup _{\|u\|_{W_{p, \pi}^{4}}=1}\left\|a_{\alpha, v}-a_{\alpha, w}\right\|_{\infty}\left\|\partial^{\alpha} u\right\|_{L_{p}} \\
& \quad \leq \sum_{|\alpha| \leq 4}\left\|a_{\alpha, v}-a_{\alpha, w}\right\|_{\infty}
\end{aligned}
$$

since $\left\|\partial^{\alpha} u\right\|_{L_{p}} \leq\|u\|_{W_{p, \pi}^{4}}=1$. We show that

$$
\left\|a_{\alpha, v}-a_{\alpha, w}\right\|_{\infty} \leq C\|v-w\|_{W_{p, \pi}^{4-4 / p}}
$$

only for $\alpha=(2,0)$, the most invloved case, as the details in other cases are similar. When $\alpha=(2,0)$ we have

$$
\begin{aligned}
\left\|a_{\alpha, v}-a_{\alpha, w}\right\|_{\infty}= & \left\|\Delta g\left((\mathrm{~A} v)^{2}\right)-\Delta g\left((\mathrm{~A} w)^{2}\right)\right\|_{\infty} \\
\leq & \sum_{j=1}^{n}\left\|g^{\prime \prime}\left((\mathrm{A} v)^{2}\right)\right\|_{\infty}\left\|\left[\partial_{x_{j}}(\mathrm{~A} v)^{2}\right]^{2}-\left[\partial_{x_{j}}(\mathrm{~A} w)^{2}\right]^{2}\right\|_{\infty} \\
& +\left\|\left[\partial_{x_{j}}(\mathrm{~A} w)^{2}\right]^{2}\right\|_{\infty}\left\|g^{\prime \prime}\left((\mathrm{A} v)^{2}\right)-g^{\prime \prime}\left((\mathrm{A} w)^{2}\right)\right\|_{\infty} \\
& +\left\|g^{\prime}\left((\mathrm{A} v)^{2}\right)\right\|_{\infty}\left\|\partial_{x_{j}}^{2}\left[(\mathrm{~A} v)^{2}-(\mathrm{A} w)^{2}\right]\right\|_{\infty} \\
& +\left\|\partial_{x_{j}}^{2}(\mathrm{~A} w)^{2}\right\|_{\infty}\left\|g^{\prime}\left((\mathrm{A} v)^{2}\right)-g^{\prime}\left((\mathrm{A} w)^{2}\right)\right\|_{\infty} \\
\leq & \sum_{j=1}^{n} C\left\|\partial_{x_{j}}\left[(\mathrm{~A} v)^{2}+(\mathrm{A} w)^{2}\right]\right\|_{\infty}\left\|\partial_{x_{j}}\left[(\mathrm{~A} v)^{2}-(\mathrm{A} w)^{2}\right]\right\|_{\infty} \\
& +C\left\|g^{\prime \prime \prime}\right\|_{\infty}\left\|(\mathrm{A} v)^{2}-(\mathrm{A} w)^{2}\right\|_{\infty} \\
& +C\left\|\partial_{x_{j}}^{2}\left[(\mathrm{~A} v)^{2}-(\mathrm{A} w)^{2}\right]\right\|_{\infty} \\
& +C\left\|_{g^{\prime \prime}}\right\|_{\infty}\left\|(\mathrm{A} v)^{2}-(\mathrm{A} w)^{2}\right\|_{\infty} \\
\leq & C \sum_{j=1}^{n} \max _{u=v, w}\left\{\left\|\partial_{x_{j}}(\mathrm{~A} u)^{2}\right\|_{\infty}\right\}\left\|\partial_{x_{j}}\left[(\mathrm{~A} v)^{2}-(\mathrm{A} w)^{2}\right]\right\|_{\infty} \\
& +\left\|\partial_{x_{j}}^{2}\left[(\mathrm{~A} v)^{2}-(\mathrm{A} w)^{2}\right]\right\|_{\infty} \\
& +\left\|(\mathrm{A} v)^{2}-(\mathrm{A} w)^{2}\right\|_{\infty} .
\end{aligned}
$$

The first inequality is obtained by computing the Laplacian and applying the triangle inequality. Since $\Omega$ is bounded, 17 and 18 imply the existence of a $C>0$ such that 


$$
\begin{aligned}
& \left\|g^{\prime \prime}\left((\mathrm{A} v)^{2}\right)\right\|_{\infty},\left\|\left[\partial_{x_{i}}(\mathrm{~A} w)^{2}\right]^{2}\right\|_{\infty}, \\
& \quad\left\|g^{\prime}\left((\mathrm{A} v)^{2}\right)\right\|_{\infty}, \text { and }\left\|\partial_{x_{i}}^{2}(\mathrm{~A} w)^{2}\right\|_{\infty} \leq C .
\end{aligned}
$$

The second and third inequalities follow from these bounds. All three terms in the last expression can be controlled by a constant times $\|v-w\|_{W_{p, \pi}^{4-4 / p}}$ by taking advantage of the facts that by 14 and 15 ,

$$
\partial_{x_{j}}^{1-\varepsilon},(-\Delta)^{1-\varepsilon} \in \mathcal{L}\left(W_{p, \pi}^{4-4 / p}, C^{2}(\bar{\Omega})\right),
$$

and that, since $U$ is bounded, there exists a constant $M_{U}<\infty$ such that

$$
M_{U}=\sup _{u \in U}\|u\|_{W_{p, \pi}^{4-4 / p}} .
$$

Indeed, for the first term, when $\mathrm{A} u=\left|\nabla^{1-\varepsilon} u\right|$, the maximum term can be bounded by

$$
\begin{aligned}
& \max _{u=v, w}\left\|\partial_{x_{j}}(\mathrm{~A} u)^{2}\right\|_{\infty}=\max _{u=v, w}\left\|\partial_{x_{j}}\left|\nabla^{1-\varepsilon} u\right|^{2}\right\|_{\infty} \\
& \quad \leq \max _{u=v, w} \sum_{k=1}^{n} 2\left\|\partial_{x_{k}}^{1-\varepsilon} u\right\|_{\infty}\left\|\partial_{x_{j}}\left(\partial_{x_{k}}^{1-\varepsilon} u\right)\right\|_{\infty} \\
& \quad \leq \max _{u=v, w} \sum_{k=1}^{n}\left\|\partial_{x_{k}}^{1-\varepsilon}\right\|_{\mathcal{L}\left(W_{p, \pi}^{4,-4}, C^{2}(\bar{\Omega})\right)}\left\|\partial_{x_{j}} \partial_{x_{k}}^{1-\varepsilon}\right\|_{\mathcal{L}\left(W_{p, \pi}^{4-4 / p}, C^{1}(\bar{\Omega})\right)}\|u\|_{W_{p, \pi}^{4-4 / p}}^{2} \\
& \quad \leq 2 C M_{U}^{2}
\end{aligned}
$$

and similarly when $\mathrm{A} u=(-\Delta)^{1-\varepsilon}$. Additionally, when $\mathrm{A}$ is the fractional Laplacian, we have

$$
\begin{aligned}
& \left.\left\|\partial_{x_{j}}^{2}\left[(\mathrm{~A} v)^{2}-(\mathrm{A} w)^{2}\right]\right\|_{\infty}=\| \partial_{x_{j}}^{2}\left[\left((-\Delta)^{1-\varepsilon} v\right)^{2}-(-\Delta)^{1-\varepsilon} w\right)^{2}\right] \|_{\infty} \\
& \quad \leq\left\|\partial_{x_{j}}^{2}\right\|_{\mathcal{L}\left(C^{2}(\bar{\Omega}), C(\bar{\Omega})\right)}\left\|(-\Delta)^{1-\varepsilon}(v+w)\right\|_{C^{2}(\bar{\Omega})}\left\|(-\Delta)^{1-\varepsilon}(v-w)\right\|_{C^{2}(\bar{\Omega})} \\
& \quad \leq 2 C\left\|(-\Delta)^{1-\varepsilon}\right\|_{\mathcal{L}\left(W_{p, \pi}^{4-4 / p}, C^{2}(\bar{\Omega})\right)}^{2} \max _{u=v, w}\left\{\|u\|_{W_{p, \pi}^{4-4 / p}}\right\}\|v-w\|_{W_{p, \pi}^{4-4 / p}} \\
& \quad \leq C M_{U}\|v-w\|_{W_{p, \pi}^{4-4 / p} .}
\end{aligned}
$$

The other necessary bounds can be found in the same manner, from which we conclude (R1).

\section{Conclusion}

Several fourth order PDEs for image denoising have been analyzed. The existence of short time solutions to two newly proposed denoising models has been proved. The proof utilizes methods of maximal regularity which allow us to handle to nonlocal nonlinearity in the proposed equations, and which are flexible enough to be applied to other fourth order equations. Sufficient criteria for applying this method to other equations have been given. 
Open Access. This article is distributed under the terms of the Creative Commons Attribution Noncommercial License which permits any noncommercial use, distribution, and reproduction in any medium, provided the original author(s) and source are credited.

\section{Appendix A. Maximal regularity}

Let $E_{1} \hookrightarrow E_{0}$ be a densely embedded pair of Banach spaces, $p \in(1, \infty)$, and $T>0$, and consider the system

$$
\left\{\begin{array}{l}
\dot{u}(t)+B u(t)=f(t), \quad \text { on }(0, T), \\
u(0)=u_{0},
\end{array}\right.
$$

where $B \in \mathcal{L}\left(E_{1}, E_{0}\right)$ and $f(t) \in L_{p}\left(0, T ; E_{0}\right)$. We call

$$
u \in W_{p}^{1}\left(0, T ; E_{0}\right) \cap L_{p}\left(0, T ; E_{1}\right)
$$

a strict solution of 20 on $[0, T]$ if $u$ satisfies 20 in the $L_{p}\left(0, T ; E_{0}\right)$ sense. If $B$ is the negative generator of an analytic semigroup and $f=0,20$ has a strict solution if and only if $u_{0}$ is in the trace space of $W_{p}^{1}\left(0, T ; E_{0}\right) \cap$ $L_{p}\left(0, T ; E_{1}\right)$, that is, if

$$
u_{0} \in\left(E_{0}, E_{1}\right)_{1-\frac{1}{p}, p}=: E_{1-\frac{1}{p}, p},
$$

where $(\cdot, \cdot)_{\theta, q}$ is the standard real interpolation functor (see [31]). That the trace space of $W_{p}^{1}\left(0, T ; E_{0}\right) \cap L_{p}\left(0, T ; E_{1}\right)$ is characterized by $E_{1-\frac{1}{p}, p}$ follows from Theorem III.4.10.2 in [2], which also gives us the embedding

$$
W_{p}^{1}\left(0, T ; E_{0}\right) \cap L_{p}\left(0, T ; E_{1}\right) \hookrightarrow C\left([0, T] ; E_{1-\frac{1}{p}, p}\right) .
$$

See [2] as well for more information about trace spaces and maximal regularity in general.

$B \in \mathcal{L}\left(E_{1}, E_{0}\right)$ is said to have the property of maximal regularity, written $B \in \mathcal{M R}_{p}\left(E_{0}, E_{1}\right)=: \mathcal{M R}_{p}$, if, for every $f \in L_{p}\left(0, T ; E_{0}\right)$ and $u_{0} \in E_{1-\frac{1}{p}, p}$, there exists a unique strict solution $u$ of 20. In this case, the Open Mapping Theorem yields the existence of an $M>0$, independent of $f$ and $u_{0}$, such that

$$
\begin{aligned}
& \|u\|_{W_{p}^{1}\left(0, T ; E_{0}\right)}+\|B u(t)\|_{L_{p}\left(0, T ; E_{0}\right)} \\
& \quad \leq M\left\{\|f(t)\|_{L_{p}\left(0, T ; E_{0}\right)}+\left\|u_{0}\right\|_{E_{1-\frac{1}{p}, p}}^{p}\right\} .
\end{aligned}
$$

The following result about nonlinear equations of a form similar to 20 , but with the operator $B$ now depending, perhaps nonlocally, on $u$, is found in [13].

Theorem 3 (Clément and $\mathrm{Li}$ ). Let $U$ be a nonempty open subset of $E_{1-\frac{1}{p}, p}$. Suppose that

$$
\begin{gathered}
A \in C^{1-}\left(U ; \mathcal{L}\left(E_{1}, E_{0}\right)\right), \\
\phi \in C^{1-, 1-}\left(\left[0, T_{0}\right] \times U, E_{0}\right),
\end{gathered}
$$


and

$$
\psi \in L_{p}\left(0, T_{0} ; E_{0}\right) .
$$

Let $u_{0} \in U$. If $A\left(u_{0}\right) \in \mathcal{M R}_{p}$, then there exist $T \in\left(0, T_{0}\right]$ and a unique function $u \in W_{p}^{1}\left(0, T ; E_{0}\right) \cap L_{p}\left(0, T ; E_{1}\right)$ satisfying

$$
\left\{\begin{array}{l}
\dot{u}(t)+A(u(t)) u(t)=\phi(t, u(t))+\psi(t) \text { on }(0, T), \\
u(0)=u_{0}
\end{array}\right.
$$

The crux of Theorem 3 is the requirement that $A\left(u_{0}\right) \in \mathcal{M R}_{p}$ for any $u_{0} \in E_{1-\frac{1}{p}, p}$. That is, existence of a solution to 24 can be found by instead studying a system of type 20 with $B=A\left(u_{0}\right)$. A well known result gives that in order for this to hold, it is necessary that $A\left(u_{0}\right)$ be the negative generator of an analytic semigroup. A sufficient condition is given by a theorem of Hieber and Prüß, which requires some preliminary definitions.

Suppose that for a domain $\Omega$, an operator $B$ generates an analytic $C_{0}$-semigroup $\mathcal{T}$ on $L_{2}(\Omega)$, where $(\Omega, \mu, d)$ is a topological space such that there exist constants $c_{1}, c_{2}>0$ for which

$$
|\mathbb{B}(x, 2 \rho)| \leq c_{1}|\mathbb{B}(x, \rho)|, \quad \text { for all } x \in \Omega, \quad \rho>0,
$$

and

$$
\operatorname{ess}_{\sup }|\mathbb{B}(x, \rho)| \leq c_{2} \operatorname{essinf}_{x \in \Omega}|\mathbb{B}(x, \rho)| .
$$

The doubling property 25 implies the existence of constants $c_{3}, \nu>0$ such that

$$
|\mathbb{B}(x, \lambda \rho)| \leq c_{3} \lambda^{\nu}|\mathbb{B}(x, \rho)|, \quad \text { for all } x \in \Omega, \quad \lambda \geq 1 .
$$

Conditions 25 and 26 are satisfied on $\mathbb{R}^{n}$ as well as on bounded subsets of $\mathbb{R}^{n}$ with Lipschitz boundary.

Suppose also that $\mathcal{T}$ may be represented as an integral operator with kernel $K(\cdot, \cdot, \cdot):(0, \infty) \times \Omega \times \Omega \rightarrow \mathbb{C}$ satisfying

$$
(\mathcal{T}(t) f)(x)=\int_{\Omega} K(t, x, y) f(y) d y
$$

for a.e. $x, t>0, f \in L_{2}(\Omega)$. $K$ is said to satisfy a Poisson bound of order $k>0$ if

$$
|K(t, x, y)| \leq\left|\mathbb{B}\left(x, t^{1 / k}\right)\right|^{-1} h\left(\frac{d(x, y)^{k}}{t}\right),
$$

for a.e. $x, y \in \Omega$ and all $t>0$, where $h$ is a bounded, decreasing, continuous and strictly positive function satisfying

$$
\lim _{r \rightarrow \infty} r^{\nu+\delta} h\left(r^{k}\right)=0
$$

for some $\delta>0$ and $\nu$ as in 27. The bounds on $K$ imply the existence of a $C_{0}$-semigroup $\mathcal{T}_{q}$ on $L_{q}(\Omega)$ for any $q \in[1, \infty)$ satisfying

$$
\mathcal{T}_{q}(t) f=\mathcal{T}(t) f, \quad \text { for } f \in L_{2}(\Omega) \cap L_{q}(\Omega), t \geq 0 .
$$

Clearly $\mathcal{T}_{q}$ may also be represented by the kernel $K$ as in 28 . Denote by $B_{q}$ the generator of $\mathcal{T}_{q}$. 
The following theorem is from [24, Theorem 3.1].

Theorem 4 (Hieber and Prüß). Let $1<p, q<\infty$, and let $(\Omega, \mu, d)$ be a topological space satisfying 25 and 26 . Let $B$ be the negative generator of an analytic $C_{0}$-semigroup $\mathcal{T}$ on $L_{2}(\Omega)$ and assume that $\mathcal{T}$ may be represented by a kernel satisfying a Poisson bound of order $k>0$. Then for each $f \in L_{p}\left(0, \infty ; L_{q}(\Omega)\right)$ there exists a unique solution

$$
u \in W_{p}^{1}\left(0, \infty ; L_{q}(\Omega)\right) \cap L_{p}\left(0, \infty ; \operatorname{Dom}\left(B_{q}\right)\right)
$$

of

$$
\left\{\begin{array}{l}
\dot{u}+B u=f, \quad t \in(0, \infty) \\
u(0)=0,
\end{array}\right.
$$

in the $L_{p}\left(0, \infty ; L_{q}(\Omega)\right)$ sense.

\section{Appendix B. Application to fourth order diffusions}

The two theorems discussed in appendix A can be applied to prove Lemma 2.

Proof of Lemma 2. Consider the abstract Cauchy problem 16. $A$ is a fourth order differential operator, so set $E_{0}=L_{p, \pi}(\Omega)=L_{p}$ and $E_{1}=W_{p, \pi}^{4}$ By the discussion in appendix $\mathrm{A}$, it is necessary to take the initial condition $u_{0}$ in the trace space $E_{1-\frac{1}{p}, p}$, which, by results in [1, Chapter 5], can be characterized by

$$
E_{1-\frac{1}{p}, p}=\left(L_{p}, W_{p, \pi}^{4}\right)_{1-\frac{1}{p}, p}=W_{p, \pi}^{4-\frac{4}{p}} .
$$

Given the choice of $E_{1}$, it is natural to consider $\mathcal{A}_{p}$, the $L_{p}$-realization of $A$, defined by

$$
\begin{aligned}
\operatorname{Dom}\left(\mathcal{A}_{p}\right) & :=W_{p, \pi}^{4} \\
\mathcal{A}_{p} f & :=A f \quad \text { for all } f \in W_{p, \pi}^{4} .
\end{aligned}
$$

In the notation of Theorem $3, \phi \equiv \psi \equiv 0$, so 22 and 23 are satisfied. Theorem 4, with the aid of Theorem 3 and the result mentioned previously regarding strict solutions to 20 when $f=0$, guarantees the existence of a time $T$ such that 16 is satisfied on the interval $(0, T)$ (that is, existence of a shorttime solution), provided there exists a nonempty open subset $U \subset E_{1-\frac{1}{p}, p}$ for which the operator $A$ satisfies the following requirements.

1. $A \in C^{1-}\left(U ; \mathcal{L}\left(W_{p, \pi}^{4}, L_{p}\right)\right)$.

2. For any $v \in U, A(v)$ is the negative generator of an analytic $C_{0}$-semigroup $\mathcal{T}$ on $L_{2}(\Omega)$, and further, $\mathcal{T}$ may be represented by a kernel satisfying a Poisson bound of order $k>0$.

Requirement 2 insures that the conditions of Theorem 4 are satisfied for the operator $A(v) . \Omega$ is bounded, so if $q \in[2, \infty)$, the generator $A_{q}$ of the semigroup $T_{q}$ discussed earlier is in fact $\mathcal{A}_{q}$, the $L_{q}$-realization of $A$. Then 
taking $q=p$, since requirement 2 insures that $-A(v)$ generates an analytic semigroup, Theorem 4 implies that for all $v \in U$,

$$
A(v) \in \mathcal{M R}_{p}\left(L_{p}, W_{p, \pi}^{4}\right)
$$

This, along with requirement 1 , ensures that the conditions for Theorem 3 are satisfied, yielding the desired short-time existence of a solution to 16 .

Requirement 1 is the same as (R1) from Lemma 2, and it is now shown that (R2) and (R3) together imply requirement 2. If (R3) holds, then the coefficients of the operator $A(v)$ are all Hölder continuous, that is, there is $\rho \in(0,1)$ such that $a_{\alpha} \in B U C^{\rho}(\Omega)$ for every $|\alpha| \leq 4$. Under these conditions, a result found in [4] gives that $A(v)$ is the negative generator of an analytic $C_{0}$-semigroup on $L_{2}(\Omega)$ if certain conditions on the principal symbol of $A(v)$ are satisfied. $A(v)$ is a fourth order linear differential operator, so it can be expressed as

$$
A\left(u_{0}\right)=\sum_{|\alpha| \leq 4} a_{\alpha} \partial^{\alpha},
$$

where $a_{\alpha}: \Omega \rightarrow \mathbb{C}$. The principal symbol of $A(v)$ is

$$
\mathcal{A}_{\pi}(x, \xi):=\sum_{|\alpha|=4} a_{\alpha}(x)(i \xi)^{\alpha}, \quad(x, \xi) \in \Omega \times \mathbb{R}^{n} .
$$

For $M>0$ and $\theta \in[0, \pi], A(v)$ is said to be uniformly $(M, \theta)$-elliptic if for all $x \in \Omega$ and $|\xi|=1$ the following conditions hold:

$$
\left\{\begin{array}{l}
\max _{|\alpha|=4}\left\|a_{\alpha}\right\|_{\infty} \leq M \\
\sigma\left(\mathcal{A}_{\pi}(x, \xi)\right) \subset\{z \in \mathbb{C} /\{0\}|| \arg (z) \mid<\theta\} \\
\left|\left[\mathcal{A}_{\pi}(x, \xi)\right]^{-1}\right| \leq M
\end{array}\right.
$$

Here $\sigma$ denotes the spectrum of an operator

$$
\sigma\left(\mathcal{A}_{\pi}(x, \xi)\right)=\mathbb{C} \backslash \rho\left(\mathcal{A}_{\pi}(x, \xi)\right)
$$

where

$$
\rho\left(\mathcal{A}_{\pi}(x, \xi)\right):=\left\{\lambda \in \mathbb{C}:\left(\lambda I-\mathcal{A}_{\pi}(x, \xi)\right) \text { is invertible }\right\}
$$

Condition (R2) requires that there is some $M>0$ and $\theta \in[0, \pi / 2)$ such that $A(v)$ is uniformly $(M, \theta)$-elliptic. A theorem by Amann, Hieber, and Simonett [4, Corollary 9.5] implies that given (R2) and (R3), $A(v)$ is the negative generator of an analytic $C_{0}$-semigroup on $L_{2}(\Omega)$. Under these conditions, [16, Theorem 9.4.2] gives that $\mathcal{T}$, the semigroup generated by $A\left(u_{0}\right)$, can be represented by a kernel with a Poisson bound of order 4 , with $h(r)=$ $a \exp \left\{-b r^{\frac{1}{3}}\right\}$ for some constants $a, b>0$. Thus, requirement 2 is satisfied if (R2) and (R3) hold, and the argument is complete. 


\section{References}

[1] Amann, H.: Nonhomogeneous linear and quasilinear elliptic and parabolic boundary value problems. Function Spaces, Differential Operators and Nonlinear Analysis, Teubner-Texte Zur Math 133, 9-126 (1993)

[2] Amann, H.: Linear and quasilinear parabolic problems. Birkhäuser, Basel (1995)

[3] Amann, H.: Time-delayed Perona-Malik problems. Acta Math. Univ. Comenianae LXXVI, 15-38 (2007)

[4] Amann, H., Hieber, M., Simonett, G.: Bounded $H_{\infty}$-calculus for elliptic operators. Differ. Int. Equ. 7(3-4), 613-653 (1994)

[5] Bai, J., Feng, X.-C.: Fractional order anisotropic diffusion for image denoising. IEEE Trans. Image Process. 16(10), 2492-2502 (2007)

[6] Bertozzi, A., Greer, J.: Low-curvature image simplifiers: global regularity of smooth solutions and Laplacian limiting schemes. Commun. Pure Appl. Math. LVII, 0764-0790 (2004)

[7] Buades, A., Coll, B., Morel, J.M.: A review of image denoising algorithms, with a new one. Multiscale Model Simulations 4(2), 490-530 (2005)

[8] Catté, F., Lions, P.-L., Morel, J.-M., Coll, T.: Image selective smoothing and edge-detection by non-linear diffusion. SIAM J. Numer. Anal 29(1), 182193 (1992)

[9] Chan, T.F., Esedoglu, S., Park, F.E.: A fourth order dual method for staircase reduction in texture extraction and image restoration problems. UCLA CAM report, 05-28, (2005)

[10] Chan, T., Marquina, A., Mulet, P.: High-order total variation-based image restoration. SIAM J. Sci. Comput, 22(2),503-516 (electronic) (2000)

[11] Charbonnier, P., Blanc-Féraud, L., Aubert, G., Barlaud, M.: Deterministic edge-preserving regularization in computed imaging. IEEE Trans. Image Process. 6, 298-311 (1997)

[12] Chiappinelli, R., Nugari, R.: The Nemitskii operators in Hölder spaces: some necessary and sufficient conditions. J. of London Math. Soc 51(2), 365-372 (1995)

[13] Clément, P., Li, S.: Abstract parabolic quasilinear equations and application to a groundwater flow problem. Adv. Math. Sci. Appl, 3 (Special Issue),17-32, $(1993 / 1994)$

[14] Didas, S., Burgeth, B., Imiya, A., Weickert, J.: Regularity and scale-space properties of fractional high order linear filtering. in scale space and pde methods in computer vision, volume 3459 of Lecture Notes in Computer Science. Springer Berlin/Heidelberg (2005)

[15] Didas, S., Weickert, J., Burgeth, B.: Properties of higher order nonlinear diffusion filtering. J. Math. Imaging Vision 35(3), 208-226 (2009) 
[16] Friedman, A.: Partial differential equations of parabolic type. Kruger, Malabar, FL (1983)

[17] Greer, J.B., Bertozzi, A.L.: Traveling wave solutions of fourth order PDEs for image processing. SIAM J. Math. Anal, 36(1),38-68 (electronic) (2004)

[18] Guidotti, P.: A new nonlocal nonlinear diffusion of image processing. J. Differ. Equ. 246(12), 4731-4742 (2009)

[19] Guidotti, P.: A new well-posed nonlinear nonlocal diffusion. Nonlinear Anal. Series A: Theory Methods Appl. 72, 4625-4637 (2010)

[20] Guidotti, P., Lambers, J.: Two new nonlinear nonlocal diffusions for noise reduction. J. Math. Imaging Vision 33(1), 25-37 (2009)

[21] Guidotti P., Longo K. Two enhanced fourth order diffusion models for image denoising. J. Math. Imaging Vision. doi:10.1007/s10851-010-0256-9

[22] Hajiaboli M.R.: A Self-governing hybrid model for noise removal. In: Advances in image and video technology, volume 5414 of Lecture notes in computer science. Springer Berlin/Heidelberg (2008)

[23] Hajiaboli, M.R.: An Anisotropic fourth-order partial differential equation for noise removal. In: Scale space and variational methods in computer vision, volume 5567 of Lecture notes in computer science. Springer Berlin/Heidelberg (2009)

[24] Hieber, M., Prüß, J.: Heat kernels and maximal $\mathrm{L}_{p}-\mathrm{L}_{q}$-estimates for parabolic evolution equations. Commun. Partial DIffer. Equ. 22, 1647-1669 (1997)

[25] Kichenassamy, S.: The Perona-Malik paradox. SIAM J. Appl. Math 57(5), 13281342 (1997)

[26] Li, F., Shen, C., Fan, J., Shen, C.: Image restoration combining a total variational filter and a fourth-order filter. J. Visual Commun. Image Represent. 18(4), 322-330 (2007)

[27] Longo, K.: Fourth order partial differential equations for image processing. $\mathrm{PhD}$ thesis, University of California, Irvine (2010)

[28] Lysaker, M., Lundervold, A., Tai, X.C.: Noise removal using fourth order differential equations with applications to medical magnetic resonance images in space-time. IEEE Trans. Image Process. 12(12), 1579-1590 (2003)

[29] Mathieu, B., Melchior, P., Outstaloup, A., Ceyral, Ch.: Fractional differentiation for edge detection. Signal Process. 83, 2421-2432 (2003)

[30] Perona, P., Malik, J.: Scale-space and edge detection using anisotropic diffusion. IEEE Trans. Pattern Anal. Machine Intel. 12, 161-192 (1990)

[31] Triebel, H.: Interpolation theory, function spaces, differential operators. North Holland, Amsterdam (1978)

[32] Tumblin, J., Turk, G.: LCIS: a boundary hierarchy for detail-preserving contrast reduction. In Proceedings of the SIGGRAPH 1999 Annual Conference on Computer Graphics, August 8-13, 1999, Los Angeles, CA, USA, Siggraph Annual 
Vol. 18 (2011) Well-posedness for a class of fourth order

Conference Series, pages 83-90. ACM Siggraph, Addison-Wesley, Longman (1999)

[33] Wei, G.: Generalized Perona-Malik equation for image restoration. IEEE Signal Process. Lett. 6(7), 165-167 (1999)

[34] Witkin, A.P.: Scale-space filtering. In: Proceedings of IJCAI, pp 1021-1019. Karlsruhe (1983)

[35] You, Y.L., Kaveh, M.: Fourth order partial differential equations for noise removal. IEEE Trans. Image Process. 9(10), 1723-1730 (2000)

P. Guidotti

Department of Mathematics,

University of California at Irvine,

Irvine, CA, USA

e-mail: gpatrick@math.uci.edu

K. Longo

Department of Mathematics,

University of California at Irvine,

Irvine, CA, USA

e-mail: rainey.katie@gmail.com

Received: 6 July 2010.

Accepted: 6 January 2011. 